\title{
Enhancement of hydroquinone efficacy in combination with EGCG on inhibition of a-MSH-dependent melanin synthesis
}

\author{
Rachel Hwang \\ Asia Pacific International School, 57 Wolgye-ro 45ga-gil, Wolgye 2(i)-dong, Nowon-gu, Seoul, 46076, Republic of Korea; \\ yjrachelhwang@gmail.com
}

\begin{abstract}
Melasma is a common pigmentation disorder, which mediates melanin synthesis and causes brown patches on the skin. Melasma treatment can be complicated because there is a broad variety of approaches with no signal standard for therapy. Melanin can be synthesized from the key enzyme tyrosinase via biosynthetic pathway. Therefore, tyrosinase inhibitors such as hydroquinone and epigallocatechin gallate (EGCG) are commonly used to treat melasma. Ultraviolet radiation (UV) is known to increase the synthesis of alpha-melanocyte stimulating hormone $(\alpha-\mathrm{MSH})$ in skin cells and mediating melanin pigmentation. For this investigation, we focused on the inhibition of $\alpha-\mathrm{MSH}$-induced melanin synthesis in mouse skin cells (B16F1) by hydroquinone and EGCG treatments. Due to the hydroquinone and EGCG's common anti-melanin synthesis property, the experiment determined whether or not this property had the potential to inhibit melanin synthesis induced by $\alpha-\mathrm{MSH}$. Prior to the quantification of melanin with absorbance measurement using a microplate reader, cytotoxicity of the hydroquinone and EGCG were determined using a fluorescence cell counter to discern whether decreases in melanin concentrations were the result of cell death. After determining the lethal dosage of each compound, B16F10 cells were treated with hydroquinone and EGCG both separately and in combination in presence of $\alpha-\mathrm{MSH}$. As a result, $10 \mu \mathrm{M}$ of hydroquinone in combination with $25 \mu \mathrm{M}$ of EGCG most effectively inhibited $\alpha-\mathrm{MSH}$-induced melanin synthesis.

KEYWORDS: Cellular and Molecular Biology; Molecular Biology; Melanin; Hydroquinone; EGCG.
\end{abstract}

\section{- Introduction}

Melanin is a light-absorbing polymer present in the animal and plant kingdoms. It is one of the primary pigments that are present in vertebrates' surface structures. The primary function of melanin is to protect the skin from ultraviolet radiation coming from the sun. This pigment is further used for commercial purposes such as a component of photoprotective creams and as a prospective target for anti-myeloma therapy. Products that make up this polymer, such as indoles, are all derived from tyrosine oxidation. ${ }^{1}$ Oxidation of tyrosine caused by the enzyme tyrosinase leads to dopa-dopaquinone couple generation, which consequently forms melanin pigments. ${ }^{2}$ Melanin is produced by melanocytes as a response to UV exposure. These melanin are transferred to neighboring keratinocytes, commonly known as skin cells, in form of melanosomes to protect the DNA from UV damage. However, when these melanocytes become hyperactive, skin may suffer the consequence of hyperpigmentation including melasma, freckles, and senile lentigines. ${ }^{3}$

Hydroquinone can be defined as a bleaching agent. About 35,000 tons of hydroquinone are manufactured annually around the world with various usages: cosmetic ingredient for skin lightening, photographic developer for black and white film, stabilizer for paints, motor oils, varnishes, etc. ${ }^{4}$ Amongst these usages, hydroquinone is used most effectively as a skin lightening ingredient in the field of dermatology and for treating disorders such as hyperpigmentation or melasma, where hydroquinone effectively inhibits enzymatic oxidation of tyrosine and its conversion to melanin by acting as a competitor for enzyme tyrosinase in presence of dopa. ${ }^{4}$ This reduction of melanin in skin cells leads to skin lightening. However, some unpropitious consequences of the usage of hydroquinone may be irritant contact dermatitis and exogenous ochronosis. These detrimental effect of hydroquinone can be explained as a result of free-radical production caused by tyrosinase-promoted phenol oxidation, which may lead to lipid peroxidation. ${ }^{2}$ Another possible explanation might be that the rapid oxidation of hydroquinone by tyrosinase induces a temporary depletion of intracellular oxygen, which may disrupt essential functions of melanocytes. ${ }^{5}$ Despite these side effects, hydroquinone is still widely used as one of the few safe skin lightening and depigmenting agents. ${ }^{4}$

To enhance the efficacy of hydroquinone's melanogenesis inhibition effect while mitigating the unwanted side effects, another compound that may relieve oxidative stress needs to be used concurrently. Previous studies have found EGCG as a potential protective agent against UVB-induced apoptosis through reducing intracellular oxidative stress. ${ }^{6-8}$ EGCG is found in green tea as a significant source of catechin, accounting for more than $50 \%$ of it. In addition, green tea also consists of epicatechin-3-gallate, epigallocatechin, epicatechin, and catechin. EGCG is a source of abundant polyphenol as well. Previous studies have shown EGCG's roles as potent antioxidant and anti-inflammatory reagent that affects proliferation, differentiation and apoptosis of skin cells. ${ }^{8}$ According to preclinical evidence derived from expanding bodies of study, EGCG has great potential to impact and cure various human diseases positively. ${ }^{9}$ 
Alpha-melanocyte stimulating hormone $(\alpha-\mathrm{MSH})$ is a major component in the response of melanocytes to ultraviolet radiation (UV) and is required for hyperpigmentation in mammals. ${ }^{10,11}$ It stimulates melanogenesis by activating microphthalmia-associated transcription factor (MITF), which is a melanocyte-specific transcription factor. This hormone can therefore be introduced to cells to mimic hyperpigmentation conditions and also can be a promising target for melanin inhibition. The objective of the experiment was to test the effect of hydroquinone and EGCG on $\alpha-\mathrm{MSH}$ dependent melanin synthesis in intracellular B16F10 cells.

\section{- Methods}

\section{Cell Culture and Maintenance:}

Frozen B16F1, which originated from the skin of a mouse, was thawed and placed into a cell culture plate with RPMI 1640 medium to maintain the cells and used for the downstream experiment. The cells were maintained in a $37^{\circ} \mathrm{C}, 5$ $\% \mathrm{CO}_{2}$ incubator. The cell media was changed every $2-3$ days after cell seeding.

\section{$\alpha-M S H$ Treatment:}

$5 \mu \mathrm{M}$ stock solution of $\alpha-\mathrm{MSH}$ was prepared with $0.2 \mathrm{mg}$ of $\alpha-\mathrm{MSH}$ and $24 \mathrm{ml}$ of deionized water. The final concentration of $0-50 \mu \mathrm{M} \alpha-\mathrm{MSH}$ was prepared and diluted in a culture medium to final concentrations and incubated for 72 $\mathrm{h}$ to synthesize melanin.

\section{Imaging of B16F1:}

Image data of melanin-producing cells were acquired after the trypsinization of cells and centrifugation in $5000 \mathrm{rpm}$ for 5 minutes. Then, cells were washed with PBS buffer solution in $1.5 \mathrm{ml}$ Eppendorf ${ }^{\mathrm{TM}}$ tubes.

\section{Treatment of Hydroquinone:}

$100 \mathrm{mM}$ of stock solution of hydroquinone was prepared by dissolving $11.1 \mathrm{mg}$ of hydroquinone in $1 \mathrm{ml}$ DMSO. The final concentration of $0-100 \mu \mathrm{M}$ was tested for downstream experiments to inhibit melanin synthesis.

\section{Treatment of EGCG:}

$100 \mathrm{mM}$ of stock solution of EGCG was prepared by dissolving $1 \mathrm{mg}$ of EGCG into DMSO.

Procedure for Forming Standard for Melanin Quantification by a Microplate Reader:

A standard melanin stock solution was made in order to be used in melanin quantification by a microplate reader. $1 \mathrm{ml}$ of $\mathrm{NaOH}$, which was used to dissolve melanin in the stock solution, was mixed with $20 \mathrm{mg}$ of melanin powder in order to make the $20 \mathrm{mg} / \mathrm{ml}$ stock solution. Varying amounts of this stock solution were put in each of the wells of the 96 well plate along with the corresponding amount of $\mathrm{NaOH}$. 7 different concentrations of the stock solution were placed in the wells $(0-500 \mu \mathrm{g} / \mathrm{ml})$. The well plate containing the standard stock solutions was put into the microplate reader in order to have its melanin quantification measured.

Procedure for Testing Microplate Reader for Measuring Melanin Quantification Based on Cell Samples:

A solution of $1 \mathrm{~N} \mathrm{NaOH}$ and $10 \%$ DMSO was mixed with each of the cell samples collected beforehand in order to break the cell walls and help melanin release. A heat block was used in order to completely melt the clumped cells into each of its solutions. When the cells were fully melted, 100 $\mu \mathrm{L}$ from each of the solutions was placed into the wells of the 96-well plate previously used. The well plate was put into the microplate reader in order for its melanin quantification to be measured. TAn absorbance of $490 \mathrm{~nm}$ was measured for all prepared samples. The results of melanin quantification based on cell samples were compared with that of the standard stock solution.

\section{Cell Viability Test:}

The cells were trypsinized at the indicated time and the percentage of viable cells was measured by counting cells, which were stained by acridine orange/propidium iodide, an apoptosis indicator, with the Luna-FL Dual Fluorescence Cell CounterTM (Logos Biosystems). This instrument is integrated with dual fluorescence microscope optics. Live and dead cells are stained with the green (live) and red (dead) fluorescence dye and labeled cell images are analyzed with integrated image analysis software.

\section{- Results and Discussion}

First, $\alpha$-MSH-dependent melanogenesis of B16F1 cell line was investigated in a conventional 2D monolayer culture environment. B16F1 cells are melanoma cell lines derived from C57BL/6 mice, which are transgenic mice that are often used to study melanin pigmentation. To quantify melanin pigmentation, a melanin concentration dependent standard line graph was generated by measuring the absorbance of cell pellet (Figure $1 \mathrm{~A}$ and $\mathrm{B}$ ). As $\alpha-\mathrm{MSH}$ concentration increased, melanin concentration increased correspondingly. It is shown that the control group ( $\alpha-\mathrm{MSH}$-free) had significantly lower melanin concentration compared to the experimental groups with 5,10, 20 , and $50 \mathrm{nM}$ of $\alpha-\mathrm{MSH}$ (Figure 1C). It could be concluded that $\alpha-\mathrm{MSH}$ successfully increases the melanin concentration in B16F1 cells after $72 \mathrm{~h}$ of treatment.
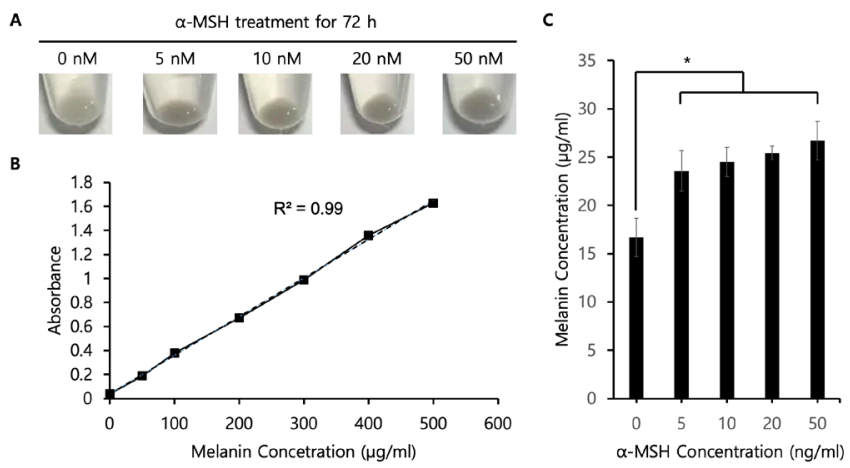

Figure 1: Effect of $\alpha$-MSH treatment on melanin synthesis in B16F1. (A) A pellet of melanocyte aggregate indicated the increase of melanin produced by $\alpha$-MSH treatment for $72 \mathrm{~h}$. (B) The standard curve was obtained from synthetic melanin to detect the concentration of melanin using $490 \mathrm{~nm}$ absorbance. (C) The amount of intracellular melanin was increased after $\alpha-\mathrm{MSH}$ treatment.

Secondly, cell cytotoxicity of hydroquinone and EGCG in various concentrations $(0-100 \mathrm{mM})$ was measured. Cell viability decreased with increasing concentrations of hydroquinone (Figure 2A). This reduction in the number of live cells became significant at $25 \mu \mathrm{M}$ of hydroquinone treatment, and the cell viability drastically decreased further with increasing con 
centrations of hydroquinone. In Figure 2B, cell viability also decreased with increasing concentrations of EGCG. However, the slope of the viability curve indicates that EGCG had a less detrimental effect on cell viability compared to hydroquinone. Reduction in cell viability was not significant until $50 \mu \mathrm{M}$ of EGCG treatment. Overall, it could be concluded that EGCG is less toxic to $\mathrm{B} 16 \mathrm{~F} 1$ cells compared to hydroquinone and that maximum of $10 \mu \mathrm{M}$ of hydroquinone and $25 \mu \mathrm{M}$ of EGCG can be used without significantly affecting cell viability.
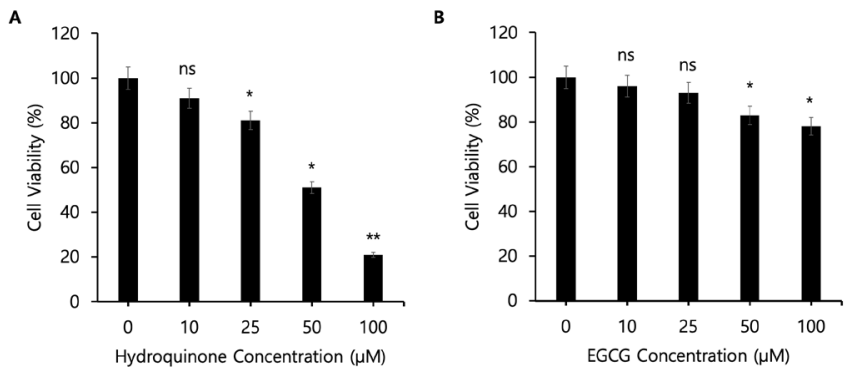

Figure 2: Significant reductions in cell viability were measured at concentrations of (A) $25 \mu \mathrm{M}$ or higher for hydroquinone and (B) $50 \mu \mathrm{M}$ or higher for EGCG. Student t-test, n=3, $0.05<$ p-value (ns), $0.05>$ p-value $\left(^{*}\right)$, $0.01>$ p-value $(* *)$.

Figure $3 \mathrm{~A}$ and $\mathrm{B}$ show the depigmenting effect of isolated and combined treatment of hydroquinone and EGCG on $\alpha-\mathrm{MSH}$-derived hyperpigmented B16F1 cells. Overall, the highest melanin concentrations were observed in B16F1 with $10 \mathrm{nM}$ of $\alpha-\mathrm{MSH}$ treatment and B16F1 with $10 \mathrm{nM}$ of $\alpha-\mathrm{MSH}$ and $25 \mu \mathrm{M}$ of EGCG treatment. The $\alpha-\mathrm{MSH}$ treatment increased the melanin concentration in the B16F1 skin cell by increasing the amount of melanin in the cell. EGCG treatment $(25 \mu \mathrm{M})$ did not seem to have a significant effect on reducing melanin synthesis in hyperpigmented cells while hydroquinone treatment $(10 \mu \mathrm{M})$ successfully reduced melanin synthesis to the level close to non-hyperpigmented cells. Furthermore, combined treatment of hydroquinone $(10 \mu \mathrm{M})$ with EGCG $(25 \mu \mathrm{M})$ most effectively reduced melanin synthesis in hyperpigmented cells to a level even below that of the non-hyperpigmented cells. This result indicates that combined treatment of hydroquinone and EGCG is more effective in inhibiting melanin synthesis compared to isolated treatments of the two compounds while minimizing the negative impact on cell viability.

The most intriguing observation of the study was the role of EGCG. When used alone, $25 \mu \mathrm{M}$ of EGCG did not exhibit any significant inhibiting effect on hyperpigmentation, but its combinatory use with hydroquinone greatly reduced melanin synthesis in hyperpigmented cells. Congruent to our initial hypothesis, this result may be explained by the oxidative stress-reducing effect of EGCG. As hydroquinone is oxidized by enzyme tyrosinase, free radicals are produced, which may lead to lipid peroxidation, resulting in damaged cell membrane. These free radicals not only negatively impact the cell's overall viability but also may reduce the activity of hydroquinone. EGCG's role as an antioxidant may relieve the symptoms of such phenomenon, which in turn, can further enhance the effectiveness of hydroquinone as a depigmenting ingredient.

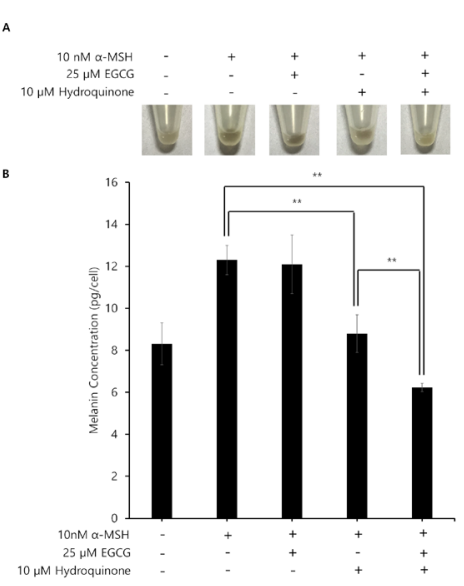

Figure 3: Effect of combined treatment of EGCG and hydroquinone on melanin synthesis. (A) A pellet of melanocyte aggregate after treatment of EGCG, hydroquinone, and both. (B) Change in melanin concentration on B16F1 after treatment of EGCG, hydroquinone, and both. Student t-test, $\mathrm{n}=3,0.01>$ p-value $\left.{ }^{(* *}\right)$.

\section{- Conclusion}

In conclusion, a combined treatment of $\alpha-\mathrm{MSH}, \mathrm{EGCG}$, and hydroquinone on B16F1 demonstrated each treatment's roles. $\alpha-\mathrm{MSH}$ treatment $(10 \mathrm{nM})$ effectively mimicked hyperpigmented melanocytes by increasing melanin synthesis in B16F1 cells, which showed significant differences in melanin concentrations from each experiment, while hydroquinone and EGCG had the effect of inhibiting melanin synthesis when used together. The most significant decrease in melanin concentrations was observed when both $25 \mu \mathrm{M}$ EGCG and $10 \mu \mathrm{M}$ hydroquinone were treated on hyperpigmented B16F1 cells. This reduction was significantly higher than when the same concentration of hydroquinone was used alone. As proposed previously, EGCG may influence melanogenesis via different pathways. One possible explanation is through down-regulation of microphthalmia-associated transcription factor (MITF), which is known to be induced by $\alpha-\mathrm{MSH}$ to increase melanogenesis. ${ }^{4}$ Also, it has been demonstrated that EGCG may reduce the detrimental effect of UVB on mTOR signaling, which is known to regulate autophagy to effectively remove damaged components in cells. ${ }^{6}$ Further studies need to be conducted to fully analyze the effectiveness of EGCG as a potential cohort of hydroquinone to maximize the efficiency of the drug.

\section{Acknowledgements}

I would like to thank Dr. Woo Rin Lee from University of Suwon for his advice and guidance for this research paper.

\section{References}

1. Rzepka, Z.; Buszman, E.; Beberok, A.; Wrześniok, D., From tyrosine to melanin: Signaling pathways and factors regulating melanogenesis. Postepy Hig Med Dosw (Online) 2016, 70 (0), 695-708

2. Palumbo, A.; d'Ischia, M.; Misuraca, G.; Prota, G., Mechanism of inhibition of melanogenesis by hydroquinone. Biochimica et Biophysica Acta (BBA) - General Subjects 1991, 1073 (1), 85-90.3. Green, J. M. The benefits of herbicide-resistant crops. Pest Manag. Sci., 2012, 68(10), 1323-1331.

3. Inoue, Y.; Hasegawa, S.; Yamada, T.; Date, Y.; Mizutani, H.; Nakata, S.; Matsunaga, K.; Akamatsu, H., Analysis of the Effects of Hydroquinone and Arbutin on the Differentiation Biological 
and Pharmaceutical Bulletin 2013, 36 (11), 1722-1730.

4. Kim, D.-S.; Park, S.-H.; Kwon, S.-B.; Li, K.; Youn, S.-W.; Park, K.-C., (-)-Epigallocatechin-3-gallate and hinokitiol reduce melanin synthesisvia decreased MITF production. Archives of Pharmacal Research 2004, 27 (3), 334.

5. Sarna, T.; Dulęba, A.; Korytowski, W.; Swartz, H., Interaction of melanin with oxygen. Archives of Biochemistry and Biophysics 1980, 200 (1), 140-148.

6. Li, C.-P.; Yao, J.; Tao, Z.-F.; Li, X.-M.; Jiang, Q.; Yan, B., Epigallocatechin-gallate (EGCG) regulates autophagy in human retinal pigment epithelial cells: A potential role for reducing UVB light-induced retinal damage. Biochemical and Biophysical Research Communications 2013, 438 (4), 739-745.

7. Kim, E.; Hwang, K.; Lee, J.; Han, S. Y.; Kim, E.-M.; Park, J.; Cho, J.Y., Skin Protective Effect of Epigallocatechin Gallate. International Journal of Molecular Sciences 2018, 19 (1), 173.

8. Cao, G.; Chen, M.; Song, Q.; Liu, Y.; Xie, L.; Han, Y.; Liu, Z.; Ji, Y.; Jiang, Q. EGCG protects against UVB-induced apoptosis via oxidative stress and the JNK1/c-Jun pathway in ARPE19 cells. Mol Med Rep 2012, 5 (1), 54-59.

9. Nordlund, J.; Grimes, P.; Ortonne, J., The safety of hydroquinone. Journal of the European Academy of Dermatology and Venereology 2006, 20 (7), 781-787.

10. Hunt, G.; Todd, C.; Cresswell, J. E.; Thody, A. J., Alphamelanocyte stimulating hormone and its analogue Nle4DPhe7 alpha-MSH affect morphology, tyrosinase activity and melanogenesis in cultured human melanocytes. Journal of Cell Science 1994, 107 (1), 205-211.

11. Hyoungseok, J.; Lim, B.; Kim, M.; Kim, Y.; Kim, W.; Ihm, C.; Noh, S.; Han, D.; Yu, H.; Choi, B., A regulatory polymorphism at position À309 in PTPRCAP is associated with susceptibility to diffuse-type gastric cancer and gene expression. Neoplasia 2009, $11,1340-1347$.

- Author

Rachel Yoojeong Hwang is currently a sophomore in Asia Pacific International School. She has great passion and interest in the field of science, especially biology. She is working towards majoring biology in the future. 